\title{
Therapie von Hand- und Fußekzemen unter stationären Bedingungen
}

\author{
Treatment of Eczema on Palms and Soles for Inpatients
}

\author{
Autoren \\ A. Reichl, V. Vögtle, G. Schiffler, L. Bröhl, R. Frommelt, U. Amon \\ Institut \\ PsoriSol-Klinik für Dermatologie \& Allergologie, Hersbruck
}

\section{Bibliografie}

Dol $10.1055 / \mathrm{s}-2007-966883$

Akt Dermatol 2007; 33:

379-384 @ Georg Thieme

Verlag KG Stuttgart · New York ISSN 0340-2541

Korrespondenzadresse

Prof. Dr. med. habil.

Ulrich Amon

Ärztlicher Direktor der

PsoriSol-Klinik für

Dermatologie \& Allergologie

Mühlstr. 31

91217 Hersbruck b. Nürnberg

amon@psorisol.de

\section{Zusammenfassung \\ $\nabla$}

Die vorliegende Studie untersucht die Therapie von Hand- und Fußekzemen bei 50 Patienten während einer stationären Krankenhausbehandlung mittels eines interdisziplinären Behandlungskonzepts. Der Therapieerfolg wurde durch die subjektive Einschätzung der Patienten anhand des Ausmaßes ihrer Lebensqualität und anhand eines objektiven Scores (Index 21) erfasst.

\section{Einleitung \\ $\nabla$}

Hand- und Fußekzeme sind meist multifaktorieller Genese und sind durch diese verschiedenen Parameter auch in ihrem Verlauf beeinflusst $[4,8]$. Die wesentlichen Ursachen für Hand- und Fußekzeme liegen vor allem in einer atopischen Diathese, Typ IV-Sensibilisierungen, irritativ-toxischen Noxen und Nikotinabusus [10,12]. Auch emotionaler Stress, Juckreiz und Kratzreaktionen können das Aufrechterhalten der Erkrankung beeinflussen [18], zudem können Hand- und Fußekzeme zu Depressionen führen [5,6]. Aufgrund der Heterogenität von Hand- und Fußekzemen mangelt es bislang an nationalen und internationalen Leitlinien.

Ziel der vorliegenden Untersuchung war die zunächst deskriptive Dokumentation einer individuellen Therapie unter kontrollierten stationären Bedingungen unter Berücksichtigung verschiedener subjektiver und objektiver Qualitätssicherungsparameter.

\footnotetext{
${ }^{*}$ Herrn Professor Dr. med. Herfried Amon, vorm. Anatomisches Institut, Philipps Universität Marburg, zum 80. Geburtstag gewidmet.
}

Die Patienten wiesen im prä- und poststationären Vergleich eine signifikante Verbesserung hinsichtlich des Hautbefundes und psychosozialer Parameter auf. Die Lebensqualität sowie der Umgang mit der Hauterkrankung konnten signifikant verbessert werden. Anhand der eingesetzten Therapiemodule wurde ein Vorschlag für einen praxisorientierten Algorithmus für Handund Fußekzeme entwickelt.

\section{Patienten und Methoden \\ $\nabla$}

Patientenkollektiv

Von Juni bis November 2005 wurde die Therapie von Hand- und Fußekzemen prospektiv bei 50 Patienten (26 Frauen, 24 Männer, Durchschnittsalter 48 Jahre), die wegen dieser Erkrankung akut-stationär in die Klinik eingewiesen wurden, dokumentiert. 40 Patienten wurden von Dermatologen eingewiesen, die übrigen von Hausärzten. $45 \%$ der Patienten wechselten prästationär wegen der betreffenden Hauterkrankung häufiger als einmal (bis zu fünfmal) ihren Hautarzt im vorangegangenen Jahr. Die durchschnittliche Verweildauer betrug $21 \pm 9,4$ Tage.

\section{Dermatologisches und verhaltens-}

therapeutisches Behandlungskonzept

Die dermatologische Therapie beinhaltete je nach Bedarf die Anwendung spezifischer Lokaltherapeutika (Tannolact ${ }^{\circledR}$ Lotio/Bad, Pandel ${ }^{\circledR}$ Salbe, Protopic ${ }^{\circledR} 0,03 \%$ bzw. 0,1\% Salbe, Elidel ${ }^{\circledR}$ Creme $1 \%$, wässrige $1 \%$-ige $\mathrm{AgNO}_{3}$-Lösung), pflegender Externa (Excipial Liposalbe ${ }^{\circledR}$, Dexpanthenol $5 \%$ in Ungt. emuls. anh.), Hautschutz (Excipial Protect ${ }^{\circledR}$, Excipial Repair $\left.{ }^{\circledR}\right)$, die Durchführung von Creme-PUVA und/oder Bade-PUVA-Therapie, Interferenzstromtherapie oder Schmierseifenbäder mit anschließender mechanischer Keratolyse. Außerdem wurde den Patienten die Bedeutung 
der Triggerfaktoren, der richtige Umgang mit der Erkrankung (z.B. Wasserkarenz) sowie das Therapiekonzept erklärt. Das verhaltenstherapeutische Konzept der Klinik beinhaltet Schulungsprogramme für Patienten zum Umgang mit chronischen Hauterkrankungen [17,26,27,32]. Diese beinhalten ErregungskontrollTrainings zur Herstellung eines ausgeglichenen vegetativen Erregungsniveaus mittels progressiver Muskelrelaxation nach Jacobson, Problemlöse-Trainings und Übungen zur sozialen Kompetenz. Außerdem werden Trainings zur Kratzkontrolle und psychologische Einzelgespräche angeboten [16].

\section{Medizinischer Fragebogen}

Es wurden Fragen zu Sozialdaten der Patienten, dem Krankheitsverlauf, bekannten Allergien (Typ I und/oder Typ IV), Nikotinabusus, prästationärer Therapie, dem Fachgebiet der Einweisers und der Häufigkeit des Hautarztwechsels im letzten Jahr abgefragt. Außerdem wurde die subjektive Einschränkung durch die Hauterkrankung und deren Ausprägung zu den Zeitpunkten T1 (bei Aufnahme) und T2 (bei Entlassung), die Teilnahmehäufigkeit an den angebotenen Trainingsprogrammen während des stationären Aufenthaltes und deren eingeschätzter Bedeutung aus Sicht der Patienten eruiert. Zum Zeitpunkt T2 wurde zusätzlich abgefragt, ob der Umgang mit der Hauterkrankung gegenüber dem Anfang verbessert sei und ob die Weiterbehandlung verstanden wurde. Des weiteren wurden die individuellen Therapieverfahren während des stationären Aufenthaltes erfragt, wobei hier in topische, systemische, Licht- und Interferenzstromtherapie unterteilt wird.

\section{Somatischer Score (Index 21)}

Der klinische Verlauf wurde durch einen somatischen Score (Index 21) bei Aufnahme und bei Entlassung dokumentiert [11,19]. Dieser dient zur Objektivierung des Schweregrades bei Handund Fußekzemen. Die Erfassung der Einzelkriterien erfolgt entweder nach einem einfachen numerischen (Bläschen, Rhagaden/ Exkoriationen pro Subregion) oder nach einem graduellen Schema (Rötung, Schuppung). Die Definition der Subregionen orientiert sich dabei an den anatomischen Strukturen. Das Scoremaximum liegt bei 21 Indexpunkten (pro Hand bzw. Fuß).

\section{Subjektive Einschätzung der Beschwerden}

Anhand von sechs Items mit einer 5-Punkte Likert-Antwortskala werden die subjektiven Einschätzungen der mit dem Hand- und Fußekzem verbundenen Beschwerden erfasst. Eingeschätzt werden die momentane Ausprägung der Hauterscheinungen, die Einschränkungen durch die Hauterkrankung, der Befall von Händen und Füßen und die Ausprägung von Juckreiz und Schlafstörungen.

\section{Statistische Auswertung}

Die Auswertung wurde mit dem Programm SPSS 11.0 für Windows durchgeführt. Mittelwertsunterschiede zwischen Präund Postmesszeitpunkten wurden mit dem t-Test für abhängige Stichproben bei Daten auf Intervallskalenniveau ermittelt. Das Signifikanzniveau wurde auf $\mathrm{p}<0.001$ (Index), $\mathrm{p}<0.01$ (Einschätzung der subjektiven Beschwerden) festgesetzt.

Es wurde bewusst auf eine kontrolliert-randomisierte Studie mit standardisierten Interventionen verzichtet, um zunächst den indikations- und stadiengerechten stationären Behandlungsverlauf der Patienten nicht zu beeinträchtigen. Um die Wirksamkeit der einzelnen Therapien beurteilen zu können, müssen weitere Studien mit Kontrollgruppen, mit standardisierten objektiven

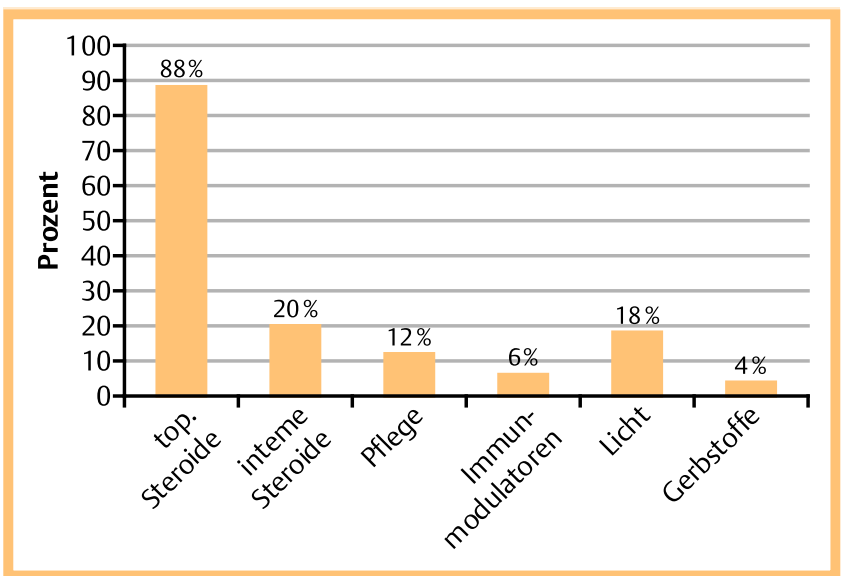

Abb. 1 Vorbehandlung bei Aufnahme.

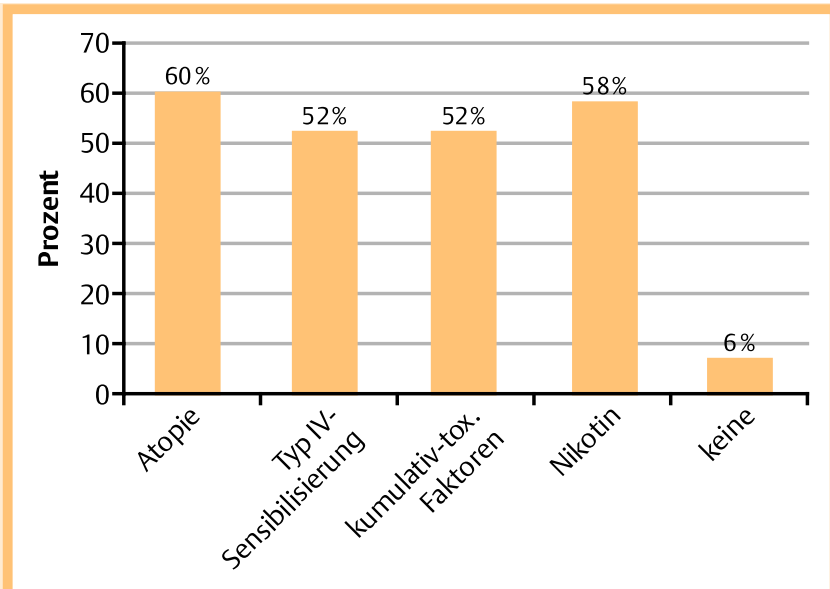

Abb. 2 Triggerfaktoren (Anamnese/Diagnostik).

Parametern, standardisierten Fragebögen usw. durchgeführt werden.

\section{Ergebnisse}

$\nabla$

56\% der Ekzeme waren bei Aufnahme hyperkeratotisch-rhagadiform, $44 \%$ dyshidrosiform. Die Vorbehandlung bis zum Aufnahmetag sowie die Auslösefaktoren der Ekzeme sind in $\bullet$ Abb. 1 u. 2 dargestellt. In $6 \%$ der Fälle ließ sich kein Trigger für die Ekzeme eruieren.

Stationär wurde bei allen Patienten eine kombinierte topische und Phototherapie durchgeführt ( $\bullet$ Abb. 3 u. 4) sowie auf eine weitgehend Steroid-sparende Anwendung geachtet. Bei $10 \mathrm{~Pa}-$ tienten war aufgrund des ausgeprägten Hautbefundes eine stadiengerechte Systemtherapie notwendig ( $\bullet$ Abb. 5). Diese wurde nach Erkennen des Nichtausreichens von konventioneller topischer Therapie eingeleitet - je nach Differenzierung (entzündlich/hyperkeratotisch). Bei Entlassung war dennoch der Hautbefund hochsignifikant verbessert ( Abb. 6).

Subjektiv beurteilten $74 \%(n=37)$ der Patienten die Ausprägung ihrer Hauterkrankung bei Aufnahme als sehr stark bzw. stark und fühlten sich durch die Hauterkrankung stark bzw. sehr stark eingeschränkt ( $\bullet$ Abb.7-9). Bei Entlassung fühlten sich noch $18 \%(n=9)$ stark eingeschränkt. Die allgemeine Belastbarkeit 


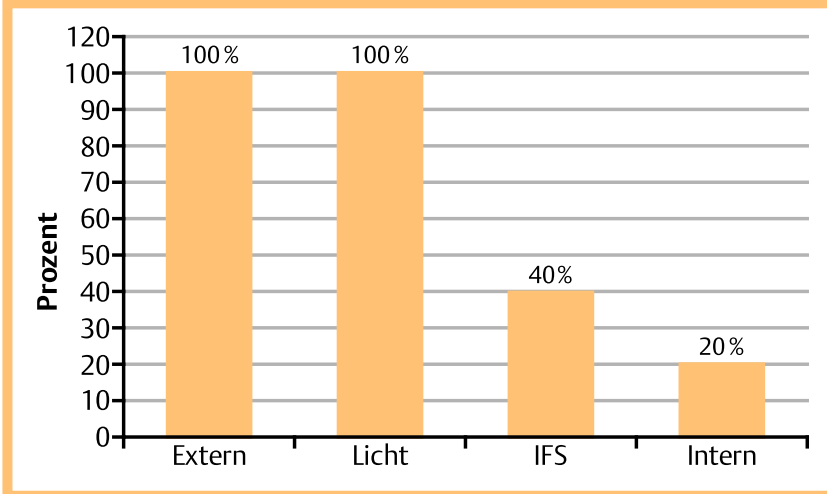

Abb. 3 Stationäre Therapie allgemein $(n=50)$.

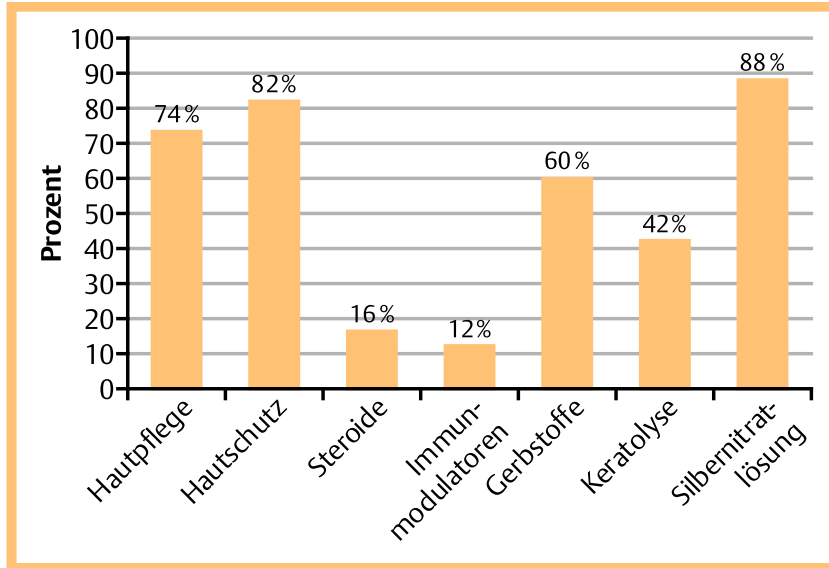

Abb. 4 Stationäre externe Behandlung $(n=50)$.

hat aus Patientensicht im Vergleich zur Aufnahme bei Entlassung deutlich zugenommen: $56 \%$ fühlten sich sehr viel stärker oder stärker belastbar als bei Aufnahme. Auch der Umgang mit der Hauterkrankung war bei Entlassung deutlich verbessert: 78\% der Patienten gaben an, dass sich der Umgang mit der Hauterkrankung „sehr stark“ oder „stark“ im Vergleich zum Aufnahmezeitpunkt verbessert habe ( $\bullet$ Abb. 7-9).

\section{Diskussion \\ $\nabla$}

Die Hände sind nicht nur als wichtige „mechanische Werkzeuge“ zu betrachten, sie sind auch ein wesentlicher Bestandteil der nonverbalen Kommunikation. Somit folgen aus Erkrankungen der Hände zum Teil Funktionseinschränkungen, bei Hautveränderungen oder sogar Deformitäten der Hände können zudem erhebliche psychische Belastungen auftreten, wie z.B. Scham, Unsicherheit, Zurückgezogenheit [4]. Auch die Füße sind für den Alltag von bedeutender Funktion. Allein der Begriff „auf eigenen Füßen stehen“ drückt die notwendige tägliche Selbstständigkeit aus. Ist unsere Fortbewegung stark behindert, sind wir auf andere angewiesen oder erheblich in unserer Lebensqualität und im Tagesablauf eingeschränkt.

Zunehmend treten seit Jahren Handekzeme als berufsbedingte Krankheiten auf [3,20,22,24]. Den Betroffenen kann dadurch Berufsunfähigkeit und damit ein Verlust der Arbeitsstelle drohen [14].

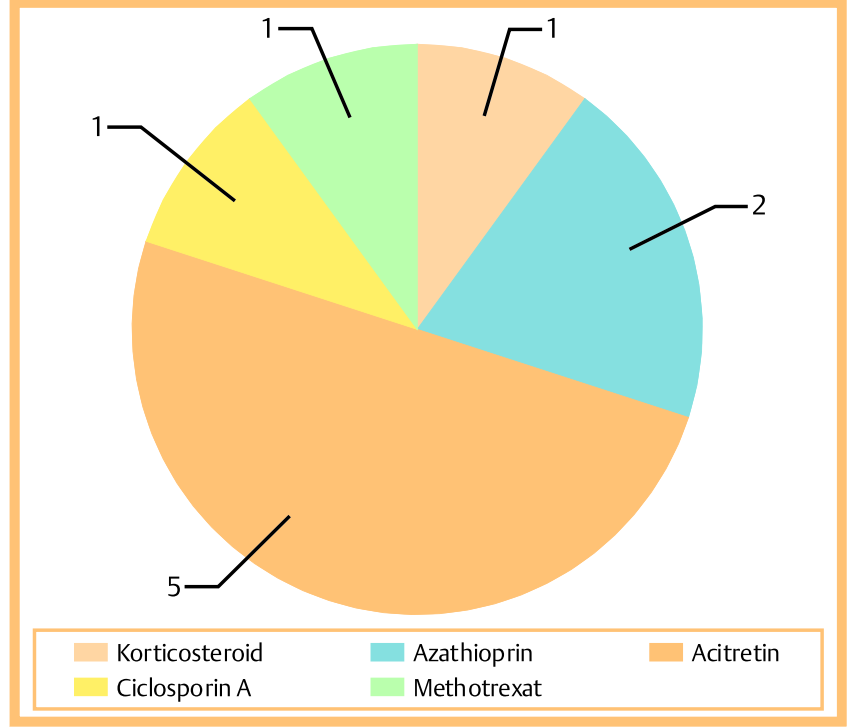

Abb. 5 Interne Behandlung $(n=10)$.

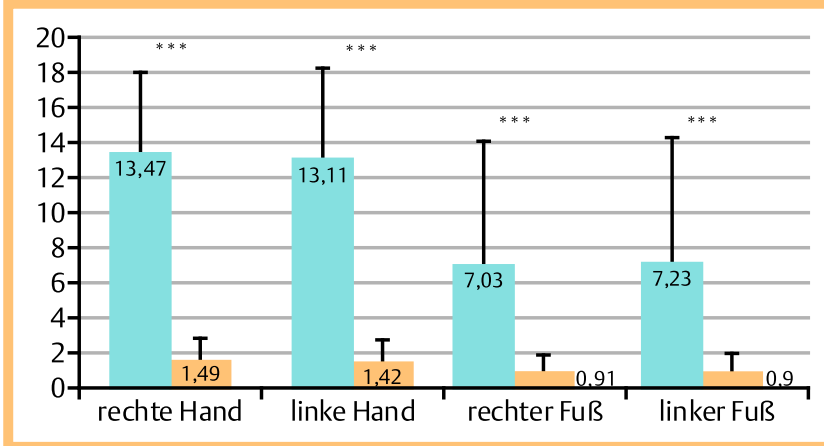

Abb. 6 Index 21 ( $\mathrm{n}=50),{ }^{* * *} \mathrm{p}<0,001$ (T1: Aufnahme, T2: Entlassung).

Berufsbedingte Hauterkrankungen sind häufig kumulativ-toxischer Genese, die tatsächlich durch den Beruf verursachten oder verschlimmerten Hauterkrankungen (BK Nr. 5101) liegen ca. 50-fach höher als die den Berufsgenossenschaften gemeldeten bzw. den versicherungsrechtlichen Voraussetzungen entsprechenden Fällen [7]. Eine entsprechende sozioökonomische Bedeutung zeigt sich in häufig sehr langen Behandlungszeiten und daraus resultierenden langen Arbeitsunfähigkeitszeiten und häufig sehr schlechter Prognose nach Tätigkeitsaufgabe [6]. Umso wichtiger ist die kontinuierliche Weiterentwicklung von langfristig wirksamen Therapiekonzepten. Aber die Therapie von Hand- und Fußekzemen ist schwierig [21]; nicht nur aufgrund der Tatsache, dass sich die verschiedenen Handekzeme in Ausprägung und Genese unterscheiden. Es gibt viele verschiedene Formen, Ursachen und Therapieansätze. Außerdem fehlen aussagekräftige klinische Studien zur Wirksamkeit der Therapien, z.B. für seit Jahren durchgeführte kortikoidhaltige Lokaltherapie oder Phototherapie $[8,28]$. Auch Leitlinien existieren weder in Deutschland, in England noch in den USA. Hand- und Fußekzeme haben einen sehr langwierigen Verlauf, außerdem ist die Therapie sehr zeitaufwendig [31]. Neuere Therapiemöglichkeiten, wie zum Beispiel der lokale Einsatz von Kalzineurininhibitoren, welche sich kurzfristig als gute Alternative für die Wechseltherapie mit lokalen Steroiden bewiesen haben [23] oder aber auch der systemische Einsatz von Biologicals, müssen 


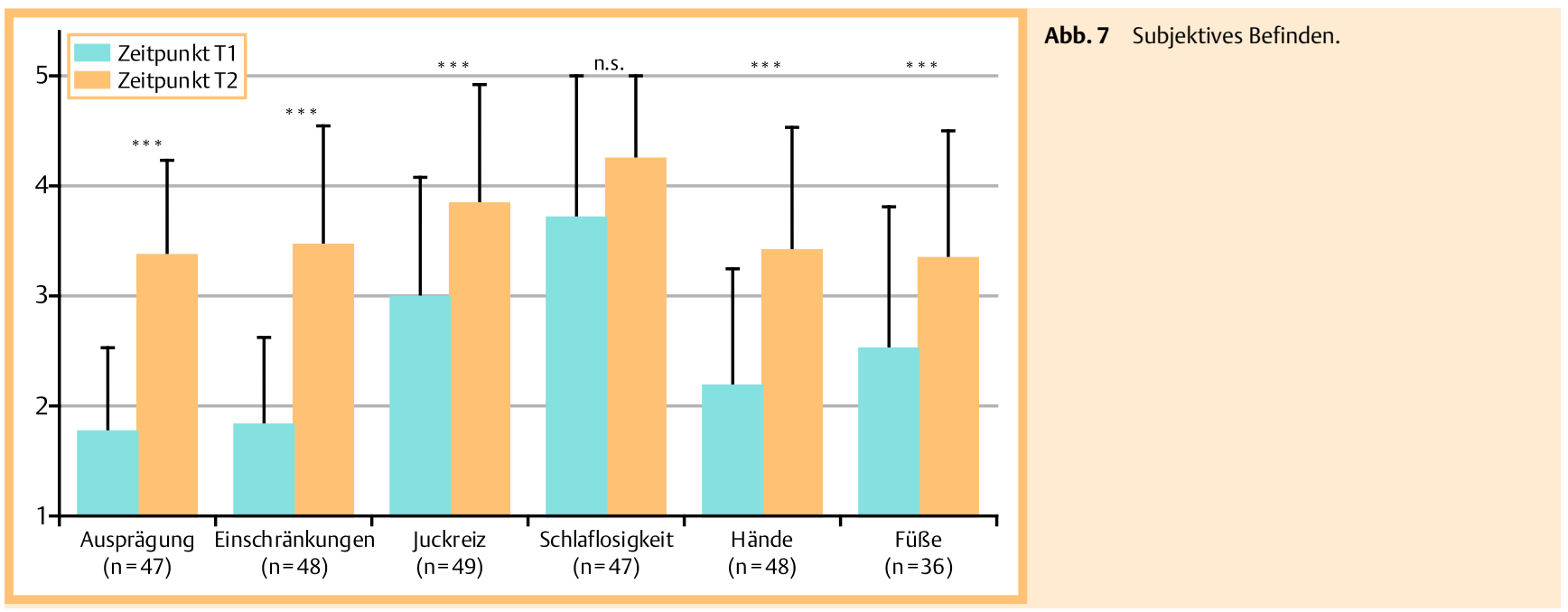

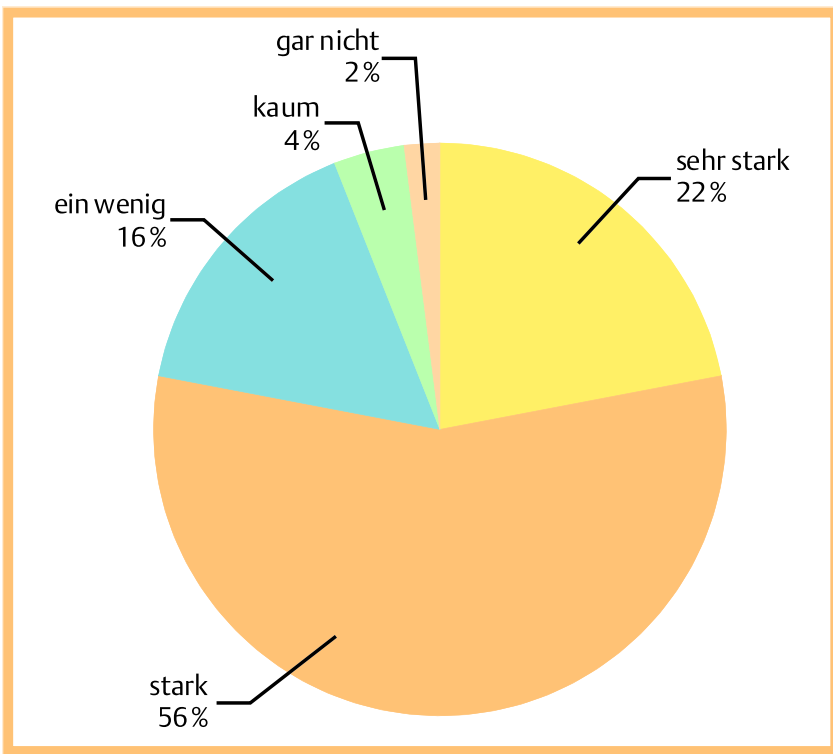

Abb. 8 Subjektive Einschätzung der Erleichterung mit dem Umgang durch den stationären Aufenthalt.

auf eine langfristige Anwendbarkeit geprüft beziehungsweise müssen langfristige Nebenwirkungen abgewartet werden.

Somit ist die Therapie derzeit eine Kombination des Wissens aus Fachbüchern, den vorhandenen teils unzureichend standardisierten Studien (welche je nach Evidenzstufen beurteilt werden sollten [8]) sowie der eigenen Erfahrung.

Einen wichtigen Pfeiler stellt zunächst die Aufklärung über die Hauterkrankung, auch ursächlich, sowie über scheinbar „banale“ Triggerfaktoren, wie z. B. Wasser, Nikotin oder Informationen zur Prophylaxe (z.B. konsequentes und adäquates Hautschutzkonzept [30], Karenz für Triggerfaktoren) dar. Auch individuelle Gegebenheiten (z. B. Typ I/IV-Sensibilisierung) müssen beachtet werden. Hier kann nicht nur die Fähigkeit sich zu sensibilisieren individuell unterschiedlich sein, sondern auch der Schweregrad der Allergie kann nach einer Sensibilisierung durch individuelle Faktoren vorgegeben sein [15]. Bei knapp 95\% der Patienten konnten ein- bis mehrere objektive Triggerfaktoren bzw. atopische Disposition nachgewiesen werden - die meist zusätzliche psychische Belastungssituation im Rahmen der Erkrankung ist als verstärkend anzusehen.

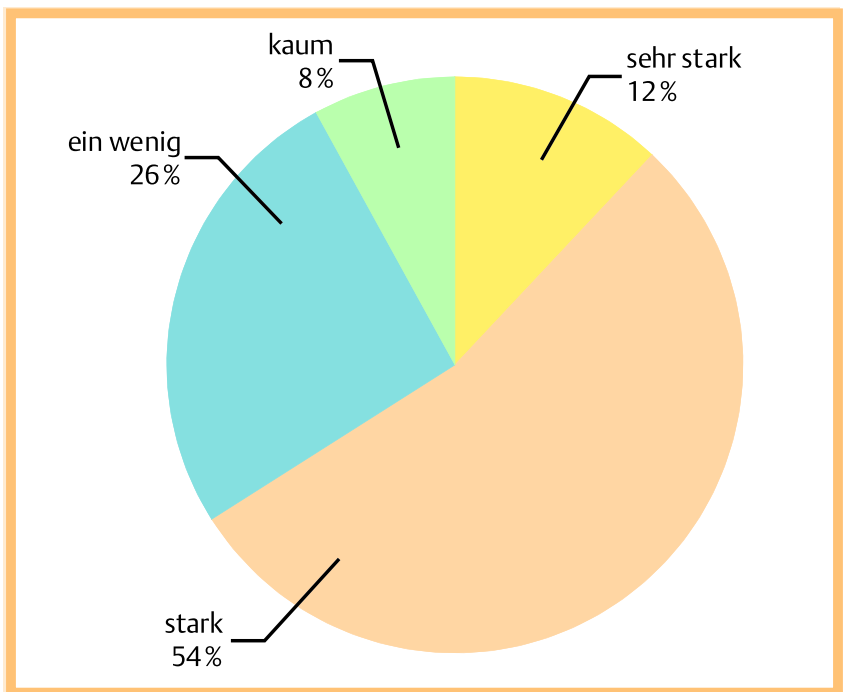

Abb.9 Subjektive Einschätzung der Belastbarkeit bei Entlassung im Vergleich zur Aufnahme.

Die Therapie richtet sich prinzipiell nach dem Status (akut-chronisch) sowie der Ätiologie des Ekzems (dyshidrosiform-rhagadiform, andere Komponente, z.B. kontaktallergisch) und kann grob in topische, systemische sowie physikalische Verfahren eingeteilt werden. Die topische Behandlung wird in kortikoidhaltige oder -freie Externa (austrocknende, desinfizierende, antibiotische/antimykotische, Vitamin $\mathrm{D}_{3}$-haltige, keratolytische, pflegende) sowie immunmodulatorische Präparate (Pimecrolimus, Tacrolimus) eingeteilt.

Systemisch stehen - je nach Akuität und Art der Hauterkrankung - unterschiedliche Behandlungsansätze zur Diskussion (z. B. Kortikoide, Antibiotika, Retinoide, Ciclosporin A, Mycophenolatmofetil, Methotrexat, Azathioprin) $[9,30]$.

Physikalische Maßnahmen beinhalten vor allem die unterschiedlichen Formen der Phototherapie, im wesentlichen Badeund/oder Creme-PUVA oder UVA1 [28 - 30] - inzwischen werden auch Therapieerfolge mit UV-freiem Licht erzielt [z. B. DermoDyne ${ }^{\circledR}$ Lichtimpfung ${ }^{\circledR}$. Salzwasser- oder Schmierseifenbäder sowie Iontophorese bzw. Interferenzstromtherapie sind ebenfalls hierzu einzuordnen. Die signifikante Verbesserung der The- 
Tab. 1 Algorithmus für die Therapie von Hand- und Fußekzemen

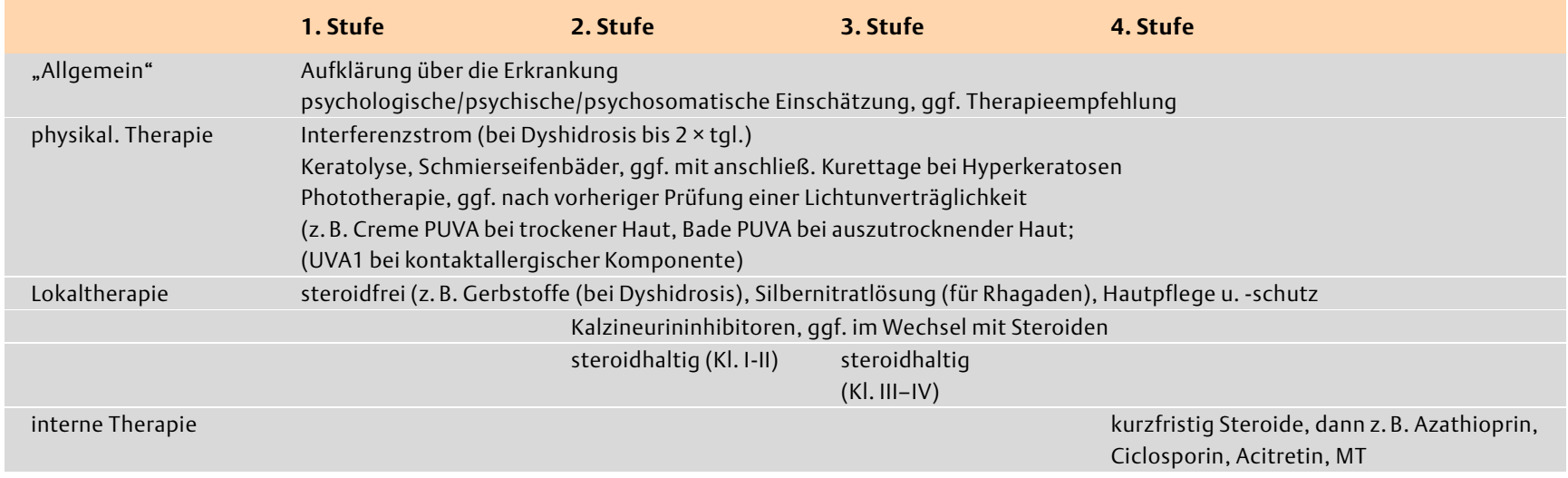

rapie durch Iontophorese bei dyshidrosiformen Handekzemen wurde in der Studie von Odia et al. belegt [19]. Eine signifikant verlängerte Rezidivfreiheit bei Patienten mit dyshidrosiformem Handekzem wurde auch in der Studie von Wollina et al. beobachtet [33].

Die in der vorliegenden Studie eingeschlossenen Patienten erhielten eine nach ihrem dermatologischen Befund, den Vorbehandlungen ausgerichtete Lokal- und Fototherapie ggf. zusätzlich eine interne Therapie sowie nach ihren Wünschen und Bedürfnissen gestaltete psychologische Therapie.

Mit einem auf die Hauterkrankung bezogenen Akuitätsscore kann durch gut objektivierbare Parameter die Ausprägung des Ekzems beurteilt werden. Nur dadurch ist ein definitiver Vergleich des Therapieerfolges, z.B. mit anderen Studien möglich [13]. Ausgehend von vorgeschlagenen Indizes, wie z.B. dem DASI, wurde in der klinischen Studie der Score „Index 21“, rein auf objektiven Kriterien beruhend, eingesetzt [11]. Hierbei konnte der INDEX 21 je an Händen und Füßen signifikant auf mindestens $11 \%$ des Ausgangswertes reduziert werden.

In der ambulanten Therapie mussten Steroide (lokal bzw. intern) aus verschiedenen Gründen sehr häufig eingesetzt werden, sämtliche andere therapeutische Maßnahmen im Vergleich hierzu nur bis zu $20 \%$.

Die stationäre Therapie bestand bei allen Patienten aus Lokal(steroidsparend) und Lichttherapie (teils allerdings nicht durchgehend bzw. wechselnd), bei $40 \%$ Interferenzstromtherapie. Immerhin bei $20 \%$ war eine interne Therapie notwendig - bei diesen stationär behandlungspflichtigen Patienten war auch eine intensive stationäre Therapie nicht ausreichend. Dennoch konnten mindestens drei Viertel der Patienten noch stationär ein Hautschutz-/pflege- Konzept erhalten - wichtig für die poststationäre Prognose ist die konkrete Empfehlung zu weiteren Behandlungen.

Die allgemeine Schwierigkeit bei der Therapie besteht darin, aus den Möglichkeiten der Therapiepalette in Abhängigkeit von Akuität/Chronizität, Ätiologie und Verlauf eine individuelle und stadiengerechte Behandlung durch Kombinationen bzw. sequentielle Verfahren durchzuführen. Per se wird wahrscheinlich für die Diagnose Hand- und/oder Fußekzem (auch in Zukunft?) aufgrund der Heterogenität keine allgemeingültige Leitlinie möglich sein.

Anhand einer ausführlichen Dokumentation als qualitätsrelevantes Merkmal kommt neben der messbaren Effektivität die subjektive Einschätzung des Behandlungserfolges von Seiten der Patienten eine wesentliche Bedeutung zu [1,2], denn die psychologische Komponente sowie die Lebensqualität sind nicht zu unterschätzende Punkte in der Dermatologie. Die subjektive Einschätzung der Ausprägung der Hauterkrankung, der Einschränkung sowie des Juckreizes waren jeweils bei Entlassung sowohl an Händen als auch an Füßen signifikant verbessert, knapp $80 \%$ der Patienten sahen durch den stationären Aufenthalt eine deutliche Erleichterung mit dem Umgang der Hauterkrankung, ein großes Interesse an Schulungen zu Hauterkrankungen signalisiert die Bedeutung des Therapiebausteins.

Der sowohl objektive als auch subjektive Behandlungserfolg kann nicht auf ein oder wenige einzelne Faktoren zurückgeführt werden, sondern ist das Ergebnis der Summe individuell eingesetzter einzelner Komponenten. Diese individuelle Vorgehensweise führte zu deutlicher Besserung des Hautbefundes sowie der Lebensqualität. Dieser Punkt muss in Zukunft für die Therapiebewertung eingefordert werden.

Somit ist im Stufen-Schema für Empfehlungen für die Praxis eine präzise Diagnostik (auch auf psychischer oder psychosomatischer Ebene) und zunächst konventionell topische, wenn möglich zunächst steroidfreie Lokaltherapie einzusetzen. Interna sollten nur bei Therapieresistenz und dann je nach Differenzierung (z.B. eher entzündlich oder eher hyperkeratotisch) angewandt werden. Ein Vorschlag für einen praxisorientierten Algorithmus wurde im Rahmen der Studie entwickelt ( Tab. 1).

Die Ergebnisse der durchgeführten Studie bestätigen die Ziele, der deutlichen Reduktion des Index 21 und der Verbesserung der Lebensqualitätsparameter unter konsequenter dermatologischer und verhaltenstherapeutischer Behandlung.

In unserer Studie wurde der Erfolg kombinierter dermatologischer und psychosozialer Interventionen auf die Verbesserung des Hautzustandes, der Kratzhäufigkeit sowie psychosozialer Parameter aufgewiesen. Es ist schwierig, den guten therapeutischen Verlauf bei einer vielschichtigen stationären Therapie auf einzelne Therapiemodule zurückzuführen. Eine deutliche Beeinflussung dieses Ergebnisses durch zusätzliche Teilnahme an verhaltenstherapeutischen Maßnahmen ist anzunehmen.

Bisher wurden nur wenige Studien zur Lebensqualität von Hand- und Fußekzemen veröffentlicht [7]. Die Lebensqualität gewinnt in der heutigen Gesellschaft jedoch zunehmend an Bedeutung. Auch in der Dermatologie sollte mehr Gewicht auf die Lebensqualität in den Studien gelegt werden [25]. 
Abstract

\section{Treatment of Eczema on Palms and Soles for Inpatients \\ $\nabla$}

The aim of the study was to investigate and document the interdisciplinary therapy for 50 patients with eczema on hands and feet during hospitalisation. Success of treatment was evaluated using subjective as well as objective measurements. The individual treatment program resulted in a significant reduction in the severity of eczema and an improvement in patients' psychosocial parameters. Quality of life was also better when patients left the clinic. The present study led to the development of an algorithm for eczema on palms and soles.

\section{Literatur}

1 Amon U, Bangha E. Qualitätsmanagement - an der Kundenzufriedenheit orientiert. T\&E Dermatol 1997; 27: 390

2 Amon U, Wenzel K, Stoll R, Fritze B, Schümann J, Bangha E, Yaguboglu R. Total Quality Management in der Dermatologie. Qualitätssicherung als obligater Bestandteil der Erfolgskontrolle bei chronisch entzündlichen Hautkrankheiten. Akt Dermatol 1997; 23: 333 - 337

3 Bauer A. Editorial: Prävention berufsbedingter Handekzeme - eine interdisziplinäre Aufgabe. Allergologie 2003; 9: 367- 368

4 Coenraads PJ, Bouma J, Diepgen TL. Lebensqualität bei Patienten mit berufsbedingten Handekzemen. Hautarzt 2004; 55: 28 - 30

5 Cvetkovski RS, Zachariae R, Jensen H, Olsen J, Johansen JD, Agner T. Quality of life and depression in a population of occupational hand eczema patients. Contact dermatitis 2006; 54: $106-111$

6 Diepgen TL. Hautkrankheiten. In: Triebig G, Kentner M, Schiele R (Hrsg). Arbeitsmedizin. Stuttgart: Gentner, 2003

7 Diepgen TL, Merk HF. Berufsbedingte Hauterkrankungen. Hautarzt 2004; 55: 8-9

8 Diepgen TL, Svensson A, Coenraads PJ. Therapie von Handekzemen. Hautarzt 2005; 56: 224-231

9 Granlund H, Erkko P, Reitamo S. Comparison of the influence od ciclosporine and topical betamethasone-17,21-diproprionate treatment on quality of life in chronic hand eczema. Avta Venerol 1997; 77: 54- 58

10 Heine G, Schnuch A, Uter W, Worm M. Type-IV-sensitization profile of individuals with atopic eczema: results from the Information Network of Departments of Dermatology (IVDK) and the German Contact Dermatitis Research Group (DKG). Allergy 2006 May; 61: 611 -616

11 Kappesser P, Mai A, Amon U. Index 21 - ein Score zur Objektivierung des Schweregrades bei Hand- und Fußekzemen. Zeitschrift für Hautkrankheiten 2001; 76 (Suppl. 1): 74

12 Krug M, Wünsche A, Blum A. Tabakabhängigkeit und die Folgen auf die Haut. Hautarzt 2004; 55: $301-315$

13 Lange S, Zschocke I, Langhardt S, Amon U, Augustin M. Effekte kombinierter therapeutischer Maßnahmen bei Patienten mit Psoriasis und atopischer Dermatitis. Hautarzt 1999; 50: 791 - 797

14 Meding B, Wrangsjo K, Jarvholm B. Fifteen-year follow-up of hand eczema: persistence and consequences. Br J Dermatol 2005; 152: $975-980$
15 Merk HF. Allergische Berufsdermatosen. Hautarzt 2004; 55: 31 - 33

16 Neumann A, Finkel D, Mundt F, Langhardt S, Bangha E, Amon U. Prozessoptimierung psychologischer Trainings in der Hautklinik im Rahmen des Total Quality Management. Präv Rehab 1998; 10: 61 -67

17 Niebel G. Verhaltensmedizin der chronischen Hautkrankheiten. Interdisziplinäre Perspektiven der atopischen Dermatitis und ihrer Behandlung. Bern: Huber, 1995

18 Niemeier V, Nippesen M, Kupfer J, Schill WB, Gieler U. Psychological factors associates with hand dermatoses: which subgroup needs additional psychological care? Br J Dermatol 2002; 146: 31 - 37

19 Odia S, Vocks E, Rakoski J, Ring J. Successful Treatment of Dyshidrotic Hand Eczema Using Tap Water Iontophoresis with Pulsed Direct Current. Acta Derm Venerol (Stockh) 1996; 76: 472 - 474

20 Orfanos CE, Garbe C. Therapie der Hautkrankheiten. 2. Aufl. Berlin: Springer, 2002

21 Przybilla B, Rueff $F$. Toxische und allergische Kontaktdermatitis. In: Braun-Falco O, Plewig G, Wolff HH (Hrsg). Dermatologie und Venerologie. Heidelberg: Springer, 2005

22 Schäfer T. Epidermiologie des beruflich bedingten Handekzems. Allergologie 2003; 9: 369-376

23 Schnopp C, Remling R, Mohrenschlager M, Weigl L, Ring J, Abeck D. Topical tacrolomus (FK506) and mometasone furoate in treatment of dyshidrotic palmar eczema: a randomized, observer-blinded trial. J Am Acad Dermatol 2002; 46: $73-77$

24 Schnuch A, Uter W, Geier J, Gefeller O. Epidemiology of contact allergy: an estimation of morbidity employing the clinical epidermiology and drug tilisation research (CE-DUR) approach. Contact Dermatitis 2002; 47: $32-39$

25 Skoet $R$, Zachariae $R$, Agner $T$. Contact dermatitis and quality of life: a structured review of the literature. Br J Dermatol. 2003; 149: 452 456

26 Stangier U, Ehlers A, Gieler U. Verhaltenstherapie und Patientenschulung bei erwachsenen Neurodermitits-Patienten.In: Petermann $\mathrm{F}$ (Hrsg)Asthma und Allergie. Verhaltensmedizinische Grundlagen und Anwendungen. 2. Aufl. Göttingen: Hogrefe, 1997

27 Stangier U, Gieler U, Ehlers A. Neurodermitis bewältigen. Heidelberg: Springer, 1996

28 Van Coevorden AM, Coenraads PJ, Svensson A et al. Overview of studies of treatments for hand eczema - the EDEN hand eczema survey. Br J Dermatol 2004; 151: 446-451

29 Van Coevorden AM, Kamphof WG, van Sonderen E, Bruynzeel DP, Coenraads $P J$. Comparison of oral psoralen-UV-A with a portable tanning unit at home vs hospital-administered bath psoralen-UV-A in patients with chronic hand eczema. Arch Dermatol 2004; 140: 1463 1466

30 Veien NK, Menne T. Treatment of hand eczema. Skn therapy Lett 2003; 5: $4-7$

31 Wallenhammar LM, Nyfjall M, Lindberg M, Meding B. Health-related quality of life and hand eczema - a comparison of two instruments, including factor analysis. J Invest Dermatol 2004; 122: 1381 - 1389

32 Warschburger P, Niebank K, Petermann F. Patientenschulung bei atopischer Dermatitis. In: Petermann F (Hrsg). Patientenschulung und Patientenberatung. Göttingen: Hogrefe, 1997

33 Wollina U, Uhlemann C, Elstermann D, Köber L, Barta U. Therapie der Hyperhidrosis mittels Leitungswasseriontophorese. Hautarzt 1998; 49: $109-113$ 